\title{
LIVRES
}

\section{Human spermatozoa in assisted reproduction}

\author{
A. A. Acosta, R. J. Swanson, S.B. Ackerman, \\ T.F. KRuger, J.A. VAN ZYL, R. MENKVE D. \\ Williams \& Wilkins , 1993, 390 pages
}

Cet ouvrage dépasse de loin un simple ouvrage de morphologie des spermatozoïdes et étudie surtout le sperme dans le cadre précis de la fécondation in vitro. Le travail a été réalisé par une cinquantaine de collaborateurs et revoit les éléments de base de la consultation d'andrologie et de la physiologie spermatique utiles au clinicien. Une large part est consacrée aux procédés de laboratoire qui sont revus en détail concernant les examens de base, les recherches d'infections spermatiques et les tests fonctionnels des spermatozoïdes. Parmi ceux-ci, l'importance de l'acrosine et de l'A.T.P. est particulièrement soulignée ainsi que le test de pénétration dans le mucus cervical et le test hyposmotique. Un chapitre est consacré à la manipulation du sperme en vue de présélection sexuelle.

L'ouvrage étant rédigé essentiellement par deux équipes, celle de Norfolk aux Etats-Unis et celle de la Ville du Cap en Afrique du Sud. L'expérience de ces deux équipes est détaillée, quant à l'évaluation et les préparations des spermatozoïdes pour fécondation in vitro. Si ce chapitre est le plus important du livre, les auteurs ne négligent pas pour autant la préparation du sperme pour insémination intra-utérine et complètent leur travail par des recherches sur le spermatozoïde en reproduction assistée, entr'autres la micro-insémination spermatique.

L'ouvrage est rédigé avec soin et les résultats des diverses approches sont illustrés par des graphiques clairs. Il s'agit d'un ouvrage qui fait étape dans tout le travail concernant le spermatozoïde dans le domaine de la fécondation in vitro et qui est un outil précieux pour tous ceux qui s'occupent de cette procédure ( $R$. Schoysman).

\section{Spermatology}

\author{
Atlas and Manual by Gerd LudWI - JUlian Frick. \\ Springer-Verlag, 1993, 160 pages
}

Les auteurs présentent un volume qui s'occupe essentiellement de l'interprétation de la morphologie des spermatozoïdes. Rédigé en langue anglaise, le premier chapitre débute par la technicité de la manipulation de l'éjaculat avec de bons détails sur les techniques de coloration. C'est essentiellement d'ailleurs sur ce problème que l'ensemble du volume porte. Le second chapitre est surtout une description détaillée des formes anormales des spermatozoïdes. Ce chapitre est illustré d'une façon très précise et abondante. Il ne s'agit pas seulement de frottis colorés, mais également d'images en microscopie à balayage. Une section importante est consacrée à l'étude des cellules rondes avec leur différenciation entre cellules de la spermatogénèse et cellules du tractus voire inflammatoires. Enfin, les tests de pénétration spermatiques dans la glaire sont également détaillés de façon précise. L'ouvrage se termine par une abondante bibliographie.

Cet ouvrage est un bon outil de travail pour tous ceux qui font des examens de laboratoire de sperme et vaut par la précision des détails techniques ainsi que par une belle et abondante iconographie (R. Schoysman). 


\section{Pratical laboratory andrology}

\author{
DAVID MORTIMER \\ Oxford University Press, 1993, 400 pages.
}

Ce manuel pratique présente les méthodes d'analyse et de traitement du sperme en usage dans les laboratoires d'andrologie. Le texte est abondamment illustré.

Principaux chapitres : Physiologie du sperme ; analyse du sperme, biochimie des spermatozoïdes et du plasma séminal; anticorps antispermatozoïdes; microbiologie et virologie du sperme, analyse du mouvement des spermatozoïdes assistée par ordinateur, ultrastructure des spermatozoïdes, évaluation du pouvoir fécondant des spermatozoïdes, application clinique des méthodes diagnostiques, lavage des spermatozoïdes, méthodes d'insémination artificielle, cryoconservation du sperme, sécurité dans les laboratoires d'andrologie, formation des techniciens, contrôle de qualité.

Cet excellent ouvrage s'adresse aux biologistes de la reproduction, aux médecins spécialistes de la stérilité, et à tous ceux qui pratiquent l'analyse et les manipulations du sperme dans les programmes de FIV et les banques de sperme (J.C. Czyba).

\section{Management and biology of carcinoma in-situ and cancer of the testis}

\author{
N. SKAKKEB FK. KM. GRIGOR, A. GIWERCMAN ET \\ M. RORTH.
}

Editions Karger, 1993, 256 pages

Cet ouvrage paru récemment représente les Actes du 3ème Workshop de Copenhague sur le carcinome in situ et le cancer testiculaire (1-4 Novembre 1992). Cet ouvrage rapporte l'ensemble des communications regroupées en 7 chapitres.

a) Pathogénèse et Biologie cellulaire du néoplasme à cellule germinale: comprend trois articles qui abordent la pathogénèse du point de vue théorique, de l'épidémiologie descriptive, de la cytogénétique moléculaire; à noter l'excellente mise au point sur la validité des dosages immunologiques des phosphatases alcalines en l'état actuel des connaissances. Conclusion sous forme de discussion générale avec résultats détaillés d'études non encore publiées.

b) Hormones et facteurs de croissance dans la néoplasie à cellule germinale. Comprend 5 articles qui traitent de l'ultrastructure des cellules germinales malignes à la culture de lignées cellulaires de tératomes humains en pas- sant par la biologie des cellules germinales primordiales et le contrôle de la prolifération cellulaire du tératome. Discussion générale à la fin.

c) Chapitre se résumant à une communication, qui est la proposition d'une nouvelle classification des tumeurs testiculaires à cellule germinale. Discussion mouvementée.

d) Ce chapitre aborde le dépistage du carcinome in situ (CIS) et la conduite à tenir. 4 articles concernant les aspects cliniques et épidémiologiques du CIS, la prévalence du CIS dans le testicule controlatéral chez des patients ayant un cancer du testicule (résultats des 2 grosses études multicentriques, et les moyens "nouveaux" de dépistage (hors biopsie testiculaire). Une discussion générale conclut bien ce chapitre en répondant à la question "Doit-on biopsier le testicule controlatéral?".

e) Ce chapitre traite des relations entre cancer du testicule et reproduction. 8 articles font le point sur les effets de la chimiothérapie, de la radiothérapie et de la chirurgie sur la spermatogénèse, la fonction gonadique, l'éjaculation, la qualité du sperme et la fertilité. Conclusion sous forme d'une discussion générale.

f) Identification et traitement des patients à haut risque. Deux temps dans ce chapitre: le premier, avec 4 articles définissant les facteurs pronostiques des tumeurs séminomateuses et non séminomateuses, se termine par une discussion générale portant sur la question suivante: "Comment reconnaître les patients ayant une tumeur testiculaire à haut risque?". Le deuxième temps porte sur différents types de traitements utilisés à l'heure actuelle.

g) Ce dernier chapitre résume les aspects cliniques du CIS du testicule, trace de nouvelles prospectives pour la biologie des tumeurs humaines à cellule germinale, et conclut sur la reconnaissance des tumeurs à haut risque et la conduite à tenir.

\section{Commentaire : ( R. Mieusset)}

Sachant que le cancer du testicule représente un quart de tous les cancers chez les hommes de 20 à 30 ans, ce bel ouvrage d'actualité, à la fois clinique, biologique et thérapeutique, mérite d'être connu par tous ceux qui s'occupent d'Andrologie. 